\title{
A Negative Biasing Experiment to Test Numerical Calculations Based on a Neoclassical L-H Transition Model in Stellarators
}

\author{
Erik N. Thogersen and J. Leon Shohet, Fellow, IEEE
}

\begin{abstract}
Numerical calculations based on Shaing's L-H transition theory in stellarators [1] and performed by Dahi et al. [2] determine viscosity as a function of ion flow speed in the interchangeable module stellarator (IMS) [3]. The calculations predict local maximums in viscosity at flows corresponding to Mach numbers $-\mathbf{2}$ and -10 , but not elsewhere. The local peaks manifest themselves as jumps in flow speed, and as regions of high radial electric field $\left(E_{r}\right)$ shear. By inducting flows swept between Mach numbers \pm 5 , an electron injection biasing probe revealed a jomp at -2 , but none at +2 , in agreement with the numerical results. A series of flow profiles at constant bias confirmed this result. Altogether, these data agree well with the numerical calculations and provide support for Shaing's L-H transition model as applied to stellarators.
\end{abstract}

\section{INTRODUCTION}

$\mathbf{T}$ THE L-H transition [4] has been induced through biasing of an electrode in the continuous current tokamak (CCT) [5], Textor [6], and Tuman-3 [7] tokamaks. It has been brought on by both positive and negative biasing, but the results are not identical. Negative biasing produced better confinement parameters in CCT and Tuman-3, while positive biasing proved better in Textor. An understanding of the role that biasing plays in the $\mathrm{L}-\mathrm{H}$ transition may be important to the design of future fusion devices.

$H$-mode occurs when shear in radial electric field, $E_{r}$, creates a barrier to particle transport, thus improving confinement properties. As ion flows are varied, with a bias probe for example, nonlinear plasma viscosity can cause a jump in $E_{r}$ and in ion flow. Since viscosity varies from magnetic surface to magnetic surface, a shear in $E_{r}$ is created as the flows at different radii undergo somewhat different jumps in $E_{r}$ and ion flow.

Shaing's model of the L-H transition predicts the nonlinear effect of viscosity on plasma flows, and has recently been extended to stellarators [1]. Numerical calculations for the in- terchangeable module stellarator (IMS) [3], based on Shaing's model, predict two regions of increased shear corresponding to a helical and toroidal peak in viscosity. The second peak appears only in stellarators, because it is caused by a lack of

Manuscript received June 6, 1996; revised August 8, 1996. This work was supported by the U.S. Department of Energy under Grant DE-FG0293ER54222.

The authors are with the Torsatron/Stellarator Laboratory, University of Wisconsin-Madison, Madison, WI 53706 USA (e-maxl: shohet@engr. wisc.edu).

Publisher Item Identifier S 0093-3813(96)08817-0. torodal symmetry. A description of the calculations and the application of Shaing's model to IMS can be found in [2].

Nonlinearity of viscosity with respect to flow speed is related to resonances with toroidal and helical variations in $B$. In IMS, numerical calculations predict local viscosity maximums for negative ion flows of Mach number $\left(M_{p}\right)$ -2 and -10 , but predict linear viscosity for positive flows [2]. (Here, $M_{p}$ is the poloidal flow velocity normalized with respect to ion thermal velocity, $M_{p}=-c E_{r} / B_{p} \nu_{t}$, with $B_{p}$ being poloidal magnetic field and $\nu_{t}$ the thermal velocity.) The local peaks are not expected to appear for negative biasing (positive flows), as they might be expected to in a larger machine, because of the effect of the normalized ion temperature and pressure gradients, $V_{p t}$ and $V_{p p}$ [2]

$$
V_{p p}=-\frac{c}{e N B_{p} \nu_{t}} \frac{d p}{d r}, \quad V_{p t}=-\frac{c}{e B_{p} \nu_{t}} \frac{d T}{d t} .
$$

Density and ion temperature gradients induce flows in the same direction as a positively biased probe, and in doing so cause asymmetry for positive/negative flow inducement. These gradients might be $\sim 0.1$ and 0.2 in a large machine, producing only a small asymmetry, but are in the range of 1.1-1.3 in IMS, where the asymmetry is more pronounced.

The presence of the tho local viscosity maximums at $M_{p}=$ -2 and -10 has been confirmed for positive biasing by Dahi et al. [2], both through sweep shots, where the bias is swept during a shot, and constant bias shots, where the bias is held constant during a shot, but changed from shot to shot.

The purpose of this paper is to determine whether such local peaks in plasma viscosity are seen for negative ion flows, or as the model predicts, they are not. An observation of linear viscosity for flows induced by negative biasing will confirm the numerical calculations, and in so doing, will provide support for the model on which those calculations are based. This model (Shaing's) might then be applied to other machines, helping either to explain current experimental results, or to predict the results of future experiments.

The functional form of viscosity can be investigated through measurements of ion flows as torque, induced through a bias probe, is varied. To see this we start with the steady-state surface-averaged poloidal momentum balance equation (see [2] for a more complete derivation)

$\left\langle B_{p} \cdot \nabla \cdot \overleftrightarrow{\pi}\right\rangle+v_{\text {eff }} N M\left\langle B_{p} \cdot \boldsymbol{V}\right\rangle+\frac{B^{\theta} B^{\zeta}}{c}\langle\boldsymbol{J} \cdot \nabla V\rangle=0$ 
Here, $\stackrel{\leftrightarrow}{\pi}$ is the viscous stress tensor, $B_{p}$ is the poloidal magnetic field, $V$ is the fluid mass flow, $\boldsymbol{J}$ is the plasma current density, $N$ and $M$ are ion density and mass, respectively, $V$ is the volume, $\nu_{\text {eff }}$ is the effective ion-neutral collision frequency (charge exchange), and $B^{\theta}$ and $B^{\zeta}$ are poloidal and toroidal contravariant components of the magnetic field, respectively. The third term in (2) is the drive term (torque due to ion orbit loss or external bias current). The first and the second terms are damping terms due to viscosity and ion-neutral collisions. In the Hamada coordinate system [8], these two terms are given by

$$
\begin{aligned}
& \frac{\left\langle\boldsymbol{B}_{p} \cdot \nabla \cdot \overleftrightarrow{\pi}\right\rangle}{N M \nu_{t}^{2} \chi^{\prime}}+v_{\text {eff }} \frac{\left\langle\boldsymbol{B}_{p} \cdot \boldsymbol{V}\right\rangle}{\nu_{t}^{2} \chi^{\prime}} \\
& =\sqrt{\pi} \sum_{-\substack{m, n=-\infty \\
m \neq 0}}^{\infty} \epsilon_{m n}^{2} m^{2}\left[I_{m n}\left(M_{p}-V_{p p}\right)+L_{m n} V_{p t}\right] \\
& \quad+v_{\text {eff }} \frac{R_{0} \epsilon^{2}}{q \nu_{t}}\left(1+2 q^{2}\right)\left(M_{p}-V_{p p}\right)
\end{aligned}
$$

where

$$
\begin{aligned}
\left\{\begin{array}{l}
I_{m n} \\
L_{m n}
\end{array}\right\}= & \frac{1}{4 \pi(m-n q)} \int_{0}^{\infty} d x x^{2} \exp (-x)\left\{\begin{array}{c}
1 \\
x-25
\end{array}\right\} \\
& \cdot \int_{-1}^{1} d y\left(1-3 y^{2}\right)^{2} \frac{c_{1}^{\prime}}{\left.y-c_{2}^{\prime}\right)^{2}+c_{1}^{\prime 2}}
\end{aligned}
$$

and

$$
c_{1}^{\prime}=\frac{v_{T}}{(m-n q) \nu \chi^{\prime} / B}, \quad c_{2}^{\prime}=\frac{m M_{p}}{(m-n q) \sqrt{x}}
$$

$m$ and $n$ are the poloidal and toroidal mode numbers, $y=$ $\nu_{l} / \nu, x=\nu^{2} / \nu_{t}^{2}$, and $q=\epsilon_{t}\left(B / B_{p}\right)$.

By inspecting (2) and (3) we can see that we have an. equation relating a driving term, bias current, with viscosity, which is given as a function of $M_{p}$. By measuring flow at different biases, we determine the relation of flow $\left(M_{p}\right)$ to bias, and by extension the dependence of viscosity on $M_{p}$.

\section{EXPERIMENTAL SETUP}

\section{A. General Experimental Description}

IMS [3] is a seven-field period, $m=7, \ell=3$ modular stellarator (major radius $(R)=0.4 \mathrm{~m}$, minor radius $(a)=0.04$ $\mathrm{m}, \imath_{0}=0.0, \imath_{a}=0.6$ ) with typical electron cyclotron heating (ECH) discharge parameters: $n_{e}=2-2.5 \times 10^{11} \mathrm{~cm}^{-3}, T_{e} \sim$ $18 \mathrm{eV}, T_{i} \sim 6 \mathrm{eV}, B_{0}=0.3 \mathrm{~T}, \mathrm{ECH}$ power $\left(P_{\mathrm{ECH}}\right)$ up to 5.5 $\mathrm{kW}$ at $9.31 \mathrm{GHz}$ with a 10 -ms pulse length. We used hydrogen gas puff pressures of 18,40 , and $164 \mu$ torr. Preionization was necessary at $18 \mu$ torr to produce plasma. Measurements of plasma electron temperature and density, plasma potential, ion temperature, and poloidal and toroidal ion flow velocities were made using a Langmuir probe, grided energy analyzer (GEA), and Mach probe, respectively. A 28-GHz microwave interferometer provided line average densities.

The Langmuir probe was used to measure both $T_{e}$ and $n_{e}$ and the plasma and floating potentials, $V_{p}$ and $V_{f}$, which in turn allow $E_{r}$, and by extension $M_{p}$, to be calculated. The choice between the $V_{p}$ and $V_{f}$ was determined by whether or not the bias on the bias probe was held constant during a shot or swept during the shot. To provide accurate readings of $V_{p}$, we swept the bias of the Langmuir over a range of $160 \mathrm{~V}$ at $1 \mathrm{KHz}$. The current and voltage signals were sorted and smoothed over a period of $3-8 \mathrm{~ms}$ so that an average $I$ versus $V$ curve could be produced. $T_{e}, N_{e}$, and $V_{p}$ could then be found by fitting an exponent and line to the curve [9]. The standard deviation of repeated measurements indicated that this method was accurate to about $\pm 1 \mathrm{~V}$, leading to an error in derived Mach number of \pm 0.8 .

Poloidal and toroidal flows were also determined using a Mach probe. Two shots were taken with the probe rotated by $180^{\circ}$ in-between shots. The flows could then be derived using routines based on the equations described by Peterson et al. [10]. We found these values to be less accurate than the flow values derived from $V_{p}$, so we used the Mach probe mainly to confirm the qualitative shape of flow profiles derived from $V_{p}$ and to look for changes in flow in the dynamic signals produced during swept bias shots. By noting the location of jumps during bias sweeps, we determined the choice of biases for constant bias shots.

Ion temperature measurements, which were necessary to run the numerical models, were performed using a grided energy analyzer (GEA). The GEA [11] consisted of four grids biased to different voltages in order to first repel electrons and then repel ions with a swept voltage. When the current measured on the last grid is compared with the repelling voltage, the energy distribution of the ions can be determined. The error in ion temperature determination was low $(\sim 1 \mathrm{eV})$ for the outer 2 $\mathrm{cm}$ of plasma, but much higher inside where the signal became very weak.

\section{B. The Biasing Probe}

We used a biasing probe located at the plasma edge to induce both positive and negative radial electric currents, and to preionize the plasma. The probe was capable of inducing both positive and negative biasing in the same shot, allowing comparison of the two flow directions without the effect of shot to shot differences. Preionization was necessary at low neutral densities (18 $\mu$ torr) in order to make plasma. The probe, pictured in Fig. 1, consisted of a $2 \times 10 \mathrm{~mm}$ iridium filament coated with thorium oxide and clamped between graphite holders which were in turn mated to copper feedthroughs. The graphite was necessary to prevent the copper feedthroughs from melting at the filament temperature. The whole assembly, except the filament, was coated with boron nitride paint to isolate it from the plasma.

A constant current, generally about $9 \mathrm{~A}$, was run through the filament to preheat it. Three seconds before a shot was taken this was increased by about $15 \%$ to bring the probe to emission temperature. The heating circuit floated relative to the machine ground, allowing the net filament bias to be controlled separately. The biasing circuit either added a constant dc bias during a shot, or was swept during the shot. The bias ranged from $\pm 300 \mathrm{~V}$ for $<1 \mathrm{~A}$ emission, to $\pm 200 \mathrm{~V}$ for $<2 \mathrm{~A}$ emission. We maintained the heating current for both positive and negative biases to provide uniform data. 


\section{Side View of Blasing Probe}

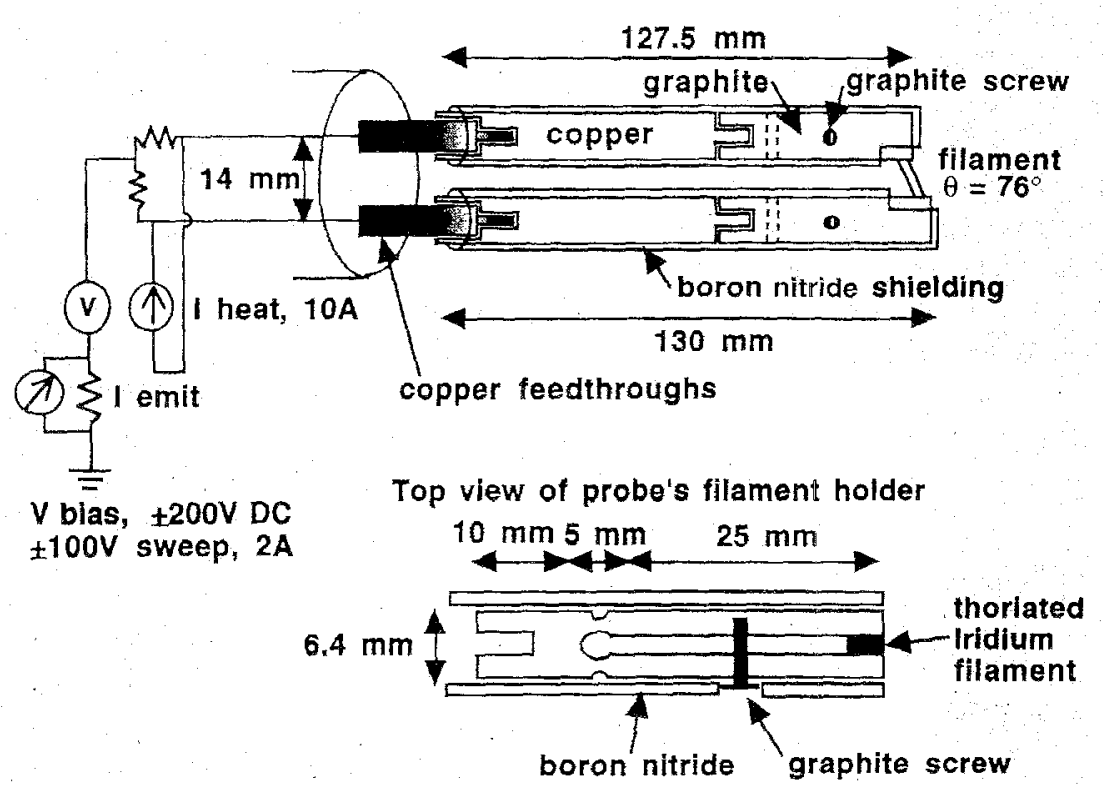

Fig. 1. The biasing probe, capable of delivering a positive or negative current of about $1 \mathrm{~A}$ and of sweeping from positive to negative during a single shot.

The probe proved capable of emitting up to about $900 \mathrm{~mA}$ with a bias of $-230 \mathrm{~V}$ and accepting $1300 \mathrm{~mA}$ at $+200 \mathrm{~V}$. We did not use higher biases in order to prevent arcing between the filament and the grounded coils or vacuum vessel. With available power supplies the probe was capable of sweeping from an emission of $+450 \mathrm{~mA}$ to $-450 \mathrm{~mA}$ in a single shot.

The initial probe design was based on lanthanum hexaboride filaments, but while $\mathrm{LaB}_{6}$ emits nicely in relatively high pressure environments (mtorr range), it did not emit at all at our working pressures ( $\sim 40 \mu$ torr), even with plasma present. Thoriated iridium filaments proved not only capable of emission at low neutral density, but were also easier to work with, being less brittle.

\section{RESULTS}

\section{A. Ion Temperature}

In order to use numerical models to predict the form of viscosity in IMS, the plasma ion temperature $\left(T_{i}\right)$ and density $\left(n_{i}\right)$ edge gradients must be known. $d n_{i}(r) / d r$ was previously measured to be $7 \times 10^{16} \mathrm{~m}^{-4}$ [12]. To determine $T_{i}(r)$ we took several profiles at eight points covering the outer $3 \mathrm{~cm}$ of plasma. These were all taken at $40 \mu$ torr, with RF powers of $5.3,3.5$, and $2.0 \mathrm{~kW}$. The profiles show a gradient of almost $2 \mathrm{eV} / \mathrm{cm}$ at the edge with an experimental error of about \pm 1 $\mathrm{eV}$. Ion temperature approximately doubled (from 3 to $7 \mathrm{eV}$ ) as RF power was raised from 2 to $5 \mathrm{~kW}$. For the numerical calculations, we set $T_{i}$ average to $6.5 \mathrm{eV}$ and $d T_{i} / d r$ to $2 \mathrm{eV} / \mathrm{cm}$ at the edge. This choice leads to normalized temperature and density gradients of 1.2 and 1.3, respectively.

\section{B. Flow Versus Bias}

Before looking for nonlinear jumps in flow, it was important to ascertain that we were indeed able to obtain large enough flows in both directions to be in the regions where such jumps might be expected to occur. We took a series of shots (at $40 \mu \mathrm{torr}, \mathrm{RF}=4 \mathrm{~kW}$ ) while varying emission current in small increments in order to determine the relationship between flow speed and emission (negative biasing) or absorption (positive biasing) current. The biasing probe was left at a constant $r=$ $41.2 \mathrm{~cm}$ and the flows were measured with a Mach probe $1 \mathrm{~mm}$ outside of the bias probe, and by taking Langmuir probe measurements of the plasma potential at 41.0 and 41.6 $\mathrm{cm}$, allowing us to estimate the radial electric field and thus determine flow speed.

Flow speed versus emission current measurements are depicted in Fig. 2. The graphs show an approximately linear dependence of flow on current between -500 and $+100 \mathrm{~mA}$ : Between +100 and $+300 \mathrm{~mA}$ there appears to be a local maximum. The flows range from Mach number -5 to +5 , demonstrating that we should be able to see the jump at $M_{p}-2$ and +2 if the latter exists. It is interesting to note that the flow's absolute magnitude actually decreases from +200 to $+400 \mathrm{~mA}$. This might seem to reflect experimental error, but it is a real effect that also appears in the Mach probe data. It can be explained by looking at flow profiles (presented later) which show that flows can decrease with increasing bias current at certain radii and for ranges of bias current corresponding to a local viscosity maximum. At the radius where the data were taken, flow actually decreases in the jump region corresponding to currents between +100 and $+300 \mathrm{~mA}$. We would expect flows to increase again beyond $+400 \mathrm{~mA}$, unfortunately we do not have data in that region to confirm this.

\section{Sweep Shots}

With the next series of shots, we sought to identify any jumps in flow as the bias was varied contintiously through a range of values. Jumps should appear as spikes in the Mach 


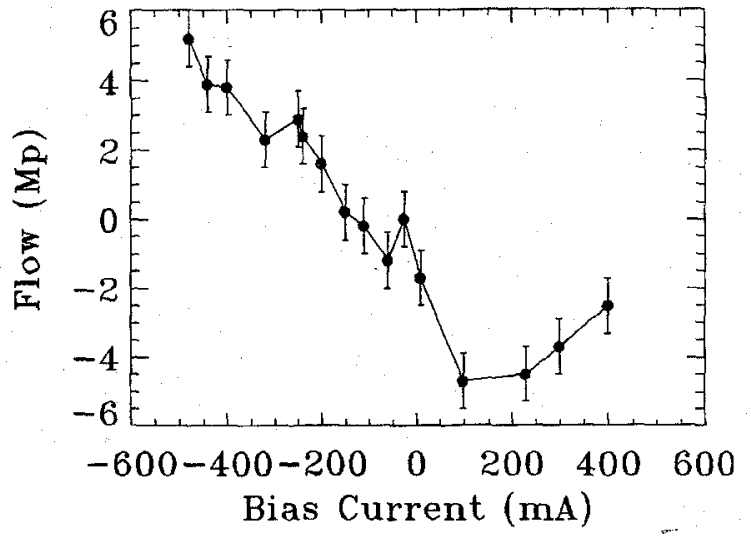

Fig. 2. Poloidal flow $\left(M_{p}\right.$ derived from $\left.E_{r}\right)$ versus bias probe current.

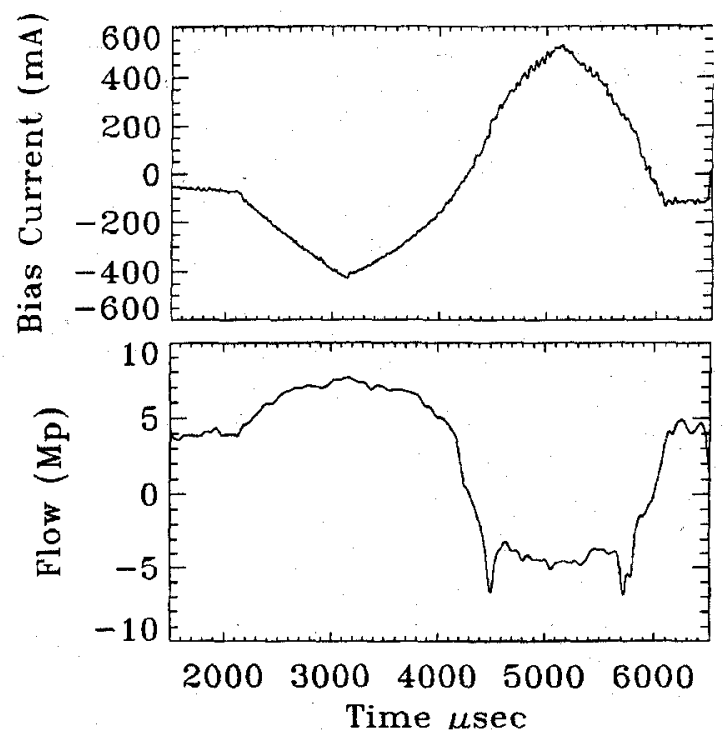

Fig. 3. Mach probe signal and bias current. Note spikes in the Mach signal beginning and ending at times corresponding to bias currents of $+100 \mathrm{~mA}$ and $+300 \mathrm{~mA}$.

probe signal, and as jumps in the dynamic floating potential signal. We swept the bias to vary the emission current from -900 to $+1300 \mathrm{~mA}$. Sample plots for the Mach probe signal, bias current, and floating potentials at two radii, focused on sweeps of $\pm 450 \mathrm{~mA}$, appear in Figs. 3 and 4 . By noting the sudden spikes in the Mach probe signal and their location on the bias current signal below, we can see only one jump in flow beginning at about $+100 \mathrm{~mA}$ and ending at $+300 \mathrm{~mA}$. This jump, which corresponds to the expected local viscosity maximum at $M_{p}=-2$, appears twice in the signal as the bias is swept through the range of nonlinear viscosity, reaches the sweep's maximum, and returns. The jump also appears in the floating potentials in Fig. 4. For positive biasing the outer floating potential is nonlinear with bias voltage while the inner is linear, indicating a jump in $E_{r}$, which is given by the difference between the potentials at two radii. Both floating potentials linearly follow the bias voltage for negative biasing, indicating no jumps for negative flows of at least Mach number +5 . This is compatible with the numerical calculations which predicted nonlinear flow for positive biasing and linear

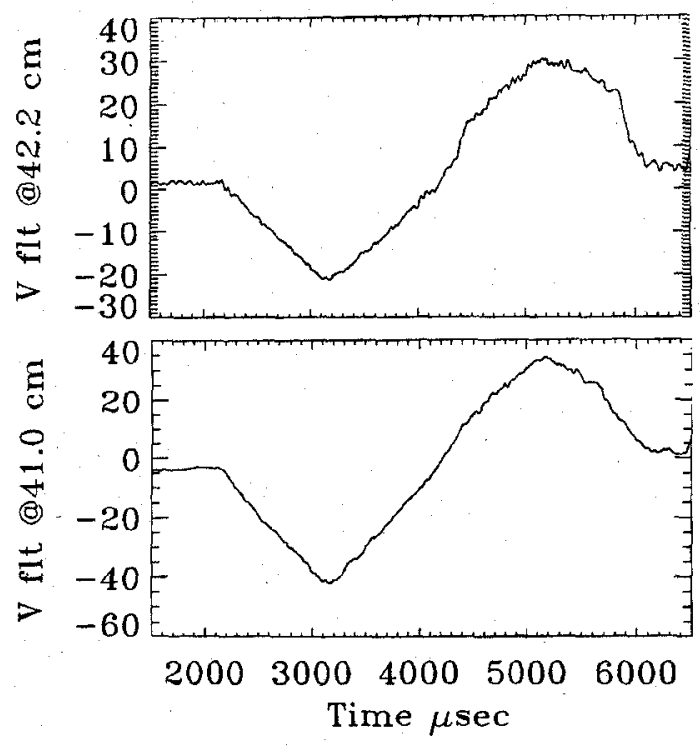

Fig. 4. Floating potentials from a Langmuir probe at two radii during a \pm 450 $m A$ sweep. Subtracting the two signals gives $E_{r}$ and thus flow. Spikes can be seen for the positive part of the sweep, but the negative half is linear.

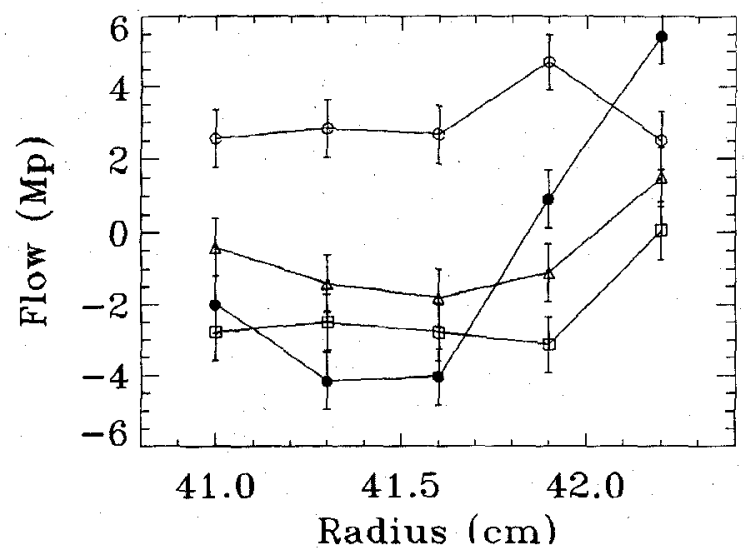

Fig. 5. Flow profiles at four different biases. Only one, corresponding to the jump at -2 , shows high shear. Filled circles represent $+200 \mathrm{~mA}$ bias, squares $+400 \mathrm{~mA}$, triangles 0 bias, and empty circles $-200 \mathrm{~mA}$.

flow for negative biasing. We do not expect to see the toroidal viscosity peak at $M_{p}=-10$ because it is very weak at the neutral density used for these shots ( $40 \mu$ torr), and because our maximum flow may not be large enough.

\section{DC Profiles}

We next took a series of flow profiles where the bias was held constant during a shot and varied only from profile to profile. Figs. 5 and 6 depict flows from $r=41.0$ to $r=42.6$ $\mathrm{cm}$ at $\mathrm{RF}=4 \mathrm{~kW}$ and a pressure of $40 \mu$ torr for a total of seven different bias currents. We note only one region with high shear in flow, i.e., a flow that changes rapidly with radius. This region (produced by bias currents of +200 and $+240 \mathrm{~mA}$ ) corresponds to the expected jump at $M_{p}=-2$. All of the other profiles are relatively flat, including the two taken at a point symmetric to the high shear region $(-200 \mathrm{~mA})$. The flatness of these profiles indicates linear flow for negative biasing, in agreement with the numerical work. If there was a jump in 


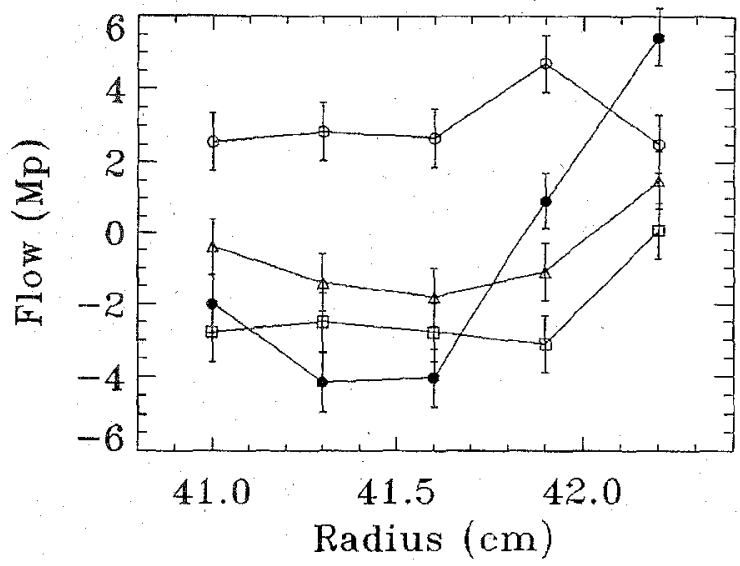

Fig. 6. Flow profiles at three other biases, confirming the result of Fig. 4. Filled circles represent $+250 \mathrm{~mA}$, empty circles $-250 \mathrm{~mA}$, and triangles 0 bias

flow at mach number +2 , it should have produced a region of high shear there.

It is also worth noting that at $r=41.3 \mathrm{~cm}$, the flow is actually greater for a bias of $+200 \mathrm{~mA}$ than it is for $+400 \mathrm{~mA}$. This is what caused the apparent decrease in flow with bias seen in Fig. 2 and described in the flow versus bias section. Had the flow versus bias measurements been taken at $r=41.9$ $\mathrm{cm}$ for example, flow would have appeared more linear for positive biasing.

\section{CONCLUSION}

Along with previously determined electron density profiles, new ion temperature gradient measurements allowed us to apply numerical models based on Shaing's L-H transition theory to IMS. Calculations performed by Dahi et al. show that viscosity should be linear in Mach number for positive flows (induced by negative biasing), and nonlinear for negative flows (induced by a positive bias), given the plasma parameters in IMS. A series of negative biasing experiments confirms this prediction. Mach probe signals recorded during swept bias shots show a single jump corresponding to the helical peak in viscosity for positive biasing. A corresponding peak is not found with negative biasing. Poloidal flow profiles, derived from plasma potentials measured with a Langmuir probe during constant bias shots, further confirm the model's predictions by revealing regions of high and low shear for positive biasing, and only low shear for negative biasing. Overall, these results agree with numerical calculations based on Shaing's L-H transition theory as applied to stellarators.

\section{ACKNOWLEDGMENT}

The authors would like to thank $\mathrm{H}$. Dahi for providing theoretical and experimental guidance for this work.

\section{REFERENCES}

[1] K. C. Shaing, "Test of tokamak L-H transition theory in stellarators," Phys. Fluids B, vol. 5, pp. 3841-3843, 1993.

[2] H. Dahi, "The nonlinear effect of viscosity on ion flow in IMS;" Ph.D. dissertation, Univ. of Wisconsin-Madison.
[3] D. T. Anderson, J. A. Derr, and J. L. Shohet, "The interchangeable module stellarator," IEEE Trans. Plasma Sci., vol. PS-9, pp. 212-220, 1981.

[4] F. Wagner ef al., "Regime of improved confinement and high beta in neutral beam heated divertor discharges;" Phys. Rey. Lett., vol. 49, pp. 1408-1412, 1982.

[5] R. J. Taylor et al., "H-mode behavior induced by cross field currents in a tokamak," Phys. Rev. Lett., vol. 63, pp. 2365-2368, 1989.

[6] R. R. Weynants et al., "H-mode behavior induced by radially outward field imposed in Textor," Plasma Phys. Contr. Nucl. Fusion, vol. 1, pp. $473-481,1990$

[7] G. L. Askinazi, V. E. Golant, S. V. Lebedev, V. A. Rozhanskij, and M. Tendler, "Radial current in a tokamak caused by a biased electrode," Nucl. Fusion, vol. 32, pp. 271-277, 1992

[8] W. D. D'haeseleer, W. N. G. Hitchon, J. D. Callen, and J. L. Shohet, Flux Coordinates and Magnetic. Field Structure. Berlin: Springer-Verlag, 1991.

[9] O. Auciello and D. L. Flamm, Eds., Plasma Diagnostics, Discharge Parameters and Chemisty. New York: Academic, 1989, ch. 3.

[10] B. J. Peterson, J. N. Talmadge, D. T. Anderson, F. S. B. Anderson, and J. L. Shohet, "Measurement of ion flows using an unmagnetized mach probe in IMS," Rev. Sci. Instrum, yol. 65, pp. 2599-2606, 1994.

[11] P. G. Matthews, "Fluctuation induced transport and poloidal rotation in IMS," Ph.D. dissertation, Univ. of Wisconsin, Madison, 1993

[12] P. G. Matthews, D. T. Anderson, F. S. B. Anderson, J. L. Shohet, and J. N. Talmadge, "Fluctuation induced transport and poloidal rotation in IMS," Phys. Fluids B, vol. 5, pp. 4061-4071, 1993.

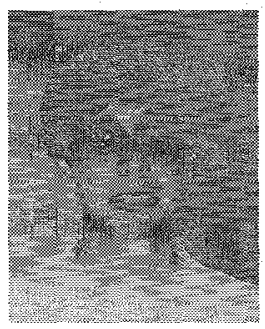

Erik N. Thogersen received the B.A. degree in physics from the University of California at Santa Cruz in 1992. He then pursued astrophysics research at the Maria Mitchell Observatory in Massachusetts and designed science exhibits for the Smithsonian's Air and Space Museum before joining the University of Wisconsin's Torsatron Stellarator Laboratory (TSL) in 1993 and beginning a master's degree in electrical engineering with a focus on plasma physics. He completed master's degree work on the interchangeable module stellarator before becoming involved with the construction of the helically symmetric experiment (HSX) at TSL.

He has moved into industry, where he is an Electrical Engineer for a motion picture special effects company.

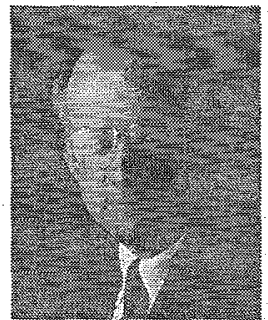

J. Leon Shohet (S'56-M'62-SM'72-F'78) received the Ph.D. degree from Carnegie Mellon University, Pittsburgh, PA, in electrical engineering in 1961.

He served on the faculty of the Johns Hopkins University, Baltimore, MD, before joining the University of Wisconsin, Madison faculty in 1966 and was appointed Professor of Electrical and Computer Engineering in 1971. He currently serves as the Director of the University's NSF Engineering Research Center for Plasma-Aided Manufacturing as well as the Director of the Torsatron/Stellarator Laboratory, a major U.S Department of Energy fusion research facility, and he is the past chairman of the Department of Electrical and Computer Engineering. He is the author of two textbooks on plasma science, over 125 journal articles, and more than 400 conference papers. He holds six patents. His research interests are: plasma-aided manufacturing; fusion, especially waves, instabilities, heating, confinement, and diagnostics; communications; magnetohydrodynamics; electromagnetic field theory; biophysics; quantum electronics; and lasers.

Dr. Shohet is a fellow of the American Physical Society. He received the Frederick Emmons Terman Award of the American Society for Engineering Education, the Merit Award of the IEEE's Nuclear and Plasma Sciences Society, the IEEE Richard F. Shea Award, the IEEE Plasma Science Prize, the IEEE Centennial Medal, and the John Yarwood Memorial Medal from the British Yacuum Council. He founded the TEEE TRANSACTIONS ON PLASMA SCIENCE in 1973. 